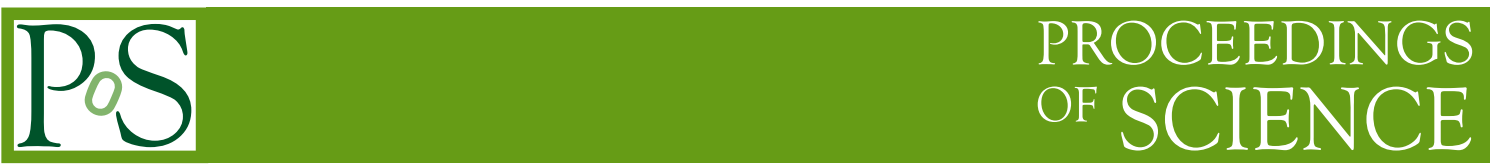

\title{
Gauge topology and confinement: an update
}

\section{Edward Shuryak*}

Department of Physics and Astronomy, Stony Brook University, Stony Brook NY 11794 USA

E-mail: edward.shuryakestonybrook. edu

In the instanton ensemble the fermionic zero modes collectivize and break chiral symmetry. Recent studies of resulting zero mode zone confirm its very small width and overall importance for lattice simulations. Confinement however has been related with completely different topological objects, the magnetic monopoles. Instanton constituents - instanton dyons, discovered at nonzero holonomy by Pierre van Baal and others - are able to explain both confinement and chiral symmetry breaking. The talk summarizes recent works deriving the instanton-dyon mutual interactions, and statistical studies of their ensemble. At high density the screening is robust enough to do it analytically, in the mean-field-type approach: we call this limit Dense Dyonic Plasma (DDP). Above $T_{c}$ the classical interaction between the dyons induce strong correlations and should be studied by direct numerical simulations. Those works are now in progress.

9th International Workshop on Critical Point and Onset of Deconfinement - CPOD2014,

17-21 November 2014

ZiF (Center of Interdisciplinary Research), University of Bielefeld, Germany

\footnotetext{
* Speaker.
} 


\section{Instantons, the Zero Mode Zone, and chiral symmetry breakings}

Instantons [1] are Euclidean 4-dimensional topological solitons of the Yang-Mills gauge fields. While small-size instantons have large action and exponentially suppressed, instantons of the size $\rho \sim 1 / 3 \mathrm{fm}$ have substantial density $n \sim 1 \mathrm{fm}^{-3}[2]$ and are therefore a very important ingredient of the gauge fields in the QCD vacuum.

Chiral anomalies induce fermionic zero modes of instantons, which generate the so called 't Hooft interaction between $2 N_{f}$ fermions, which explicitly violates the $U_{A}(1)$ chiral symmetry. Statistical mechanics of instanton ensemble, including 't Hooft interaction to all orders, known as the Interacting Instanton Liquid Model, has been solved numerically, by brute force, two decades ago, for a review see [3]. Not going into details, we remind the central concept crucial for understanding of the spontaneous $S U\left(N_{f}\right)$ chiral symmetry breaking: a collectivization of these fermionic zero modes into the so called Zero Mode Zone (ZMZ) of quasi-zero Dirac eigenstates. When those have a finite density at zero eigenvalues $n(\lambda \rightarrow 0) \neq 0$, quark condensate appears. What was known for decades (and few times rediscovered by lattice practitioners) is that the diluteness of topological ensembles leads to small "quark hopping" matrix elements of the Dirac operator, between zero modes of different solitons $(\mathrm{i}, \mathrm{j})$. In the instanton liquid with 4-density $n$ and size $\rho$ they are of the order of

$$
\sigma \sim|<i| D|j>| \sim \rho^{2} n^{1 / 2} \sim 20 \mathrm{MeV}
$$

and so is the characteristic width of the $\mathrm{ZMZ}{ }^{1}$. While the $\mathrm{ZMZ}$ states, with Dirac eigenvalues $|\lambda|<\sigma \sim 20 \mathrm{MeV}$, make only tiny $\left(\sim 10^{-4}\right)$ subset of all fermionic states on the lattice, they are responsible for a significant fraction of most hadronic masses. Studies of ZMZ states, in the context of the instanton liquid and lattice, has been made in 1990's. Recently Graz group [10] had, once again, demonstrated its importance by removing all the $\mathrm{ZMZ}$ states from the fermionic propagators: they observe a completely different hadronic spectrum, in which both of the $U_{A}(1)$ and the $S U\left(N_{f}\right)$ chiral symmetry breaking is gone. So, gauge topology is not just some cute little effect which some theory-inclined people tend to study: it is in fact responsible for a significant fraction of hadronic masses (and thus our own weight)!

Another feature observed by the Graz group is strong reduction of the statistical fluctuations in the ensemble, which is of practical importantance for lattice community. In spite of many millions of lattice variables it is hardly surprising, since there are only about a dozen or so instantons in the box. Let me predict that current efforts to improve efficiency of lattice updates using multi-grid algorithms for small Dirac eigenvalues are bound to eventually rediscover the instanton ensemble, with a delay of 3 decades or so.

At the end of this introductory section, let me mention progress in application of instantons in other directions. Instanton-induced t' Hooft interaction generates attraction between quarks, well explaining the "scalar diquark" phenomenon well known in hadronic phenomenology. Scalar diquarks become Cooper pairs of color superconductivity at high density [4]. Instantons also generate important spin effects in hadronic reactions, such as spin asymmetries [5].

\footnotetext{
${ }^{1}$ Incidentally, it is of the order of quark masses used on the lattice in the previous decades: therefore simple linear/log expressions of chiral perturbation theory did not work well.
} 
On a more theoretical note, let me remind that the Seiberg-Witten solution of $\mathscr{N}=2$ supersymmetric gluodynamics, based on the celebrated elliptic curve, was derived by the explicit calculation of all instanton amplitudes [6]. Recently [7] it has been further related to instantons in some quantum-mechanical systems.

There is also recent progress in the latter field, fueled by exact global boundary condition relating ordinary perturbation theory and those on top of the instantons, pioneered by Zinn-Justin. Dunne and Unsal [9] have suggested even more direct relation between them: however its meaning remains unclear. Our modest contribution to that development was explicit calculation of the threeloop corrections to the instanton density [8] by the direct evaluation of all Feynman diagrams: the result is consistent with the relations mentioned.

\section{The instanton-dyons and confinement}

Semiclassical theory of instantons is parametrically good at high $T$, as the effective coupling gets there weak: but they are there strongly suppressed. When light fermions are present in the theory, at high $T$ the "instanton liquid" is absent, and instantons can exist (i) either in form of neutral instanton-antiinstanton molecules [15], or (ii) single instantons, with the density proportional to all quark masses $\Pi_{f} m_{f}$, as argued by 't Hooft in his original paper on fermion zero modes. Since even the unphysical quark masses used in lattice simulations are still rather small, only recently the Bielefeld groups [16] had clearly identified a contribution in the Dirac eigenvalue density of the kind $n(\lambda) \sim m^{2} \delta(\lambda)$, corresponding to single instantons.

Decreasing the temperature below $2 T_{c}$ one finds a nontrivial average value of the Polyakov line $\left\langle P>\neq 1\right.$, indicating that an expectation value of the gauge potential is nonzero $\left\langle A_{4}\right\rangle=$ $v \neq 0$. This calls for re-defining the boundary condition of $A_{4}$ at infinity, for all solitons including instantons. That lead to 1998 discovery $[11,12]$ that nonzero $v$ splits instantons into $N_{c}$ (number of colors) constituents, the selfdual instanton-dyons ${ }^{2}$. Since these objects have nonzero electric and magnetic charges and source Abelian (diagonal) massless gluons, the corresponding ensemble is an "instanton-dyon plasma", with long-range Coulomb-like forces between constituents. By tradition the selfdual ones are called $M$ with charges $(e, m)=(+,+)$ and $L$ with charges $(e, m)=(-,-)$, the anti-selfdual antidyons are called $\bar{M},(e, m)=(+,-)$ and $\bar{L},(e, m)=(-,+)$.

Diakonov and collaborators (for review see [20] ) emphasized that, unlike the (topologically protected) instantons, the dyons interact directly with the holonomy field. They suggested that since such dyon (anti-dyon) become denser at low temperature, their back reaction may overcome perturbative holonomy potential and drive it to its confining value, leading to vanishing of the mean Polyakov line, or confinement.

Specifically, Diakonov and collaborators focused on the self-dual sector $L, M$ and studied the one-loop contribution to the partition function [19]. The volume element of the moduli space was written in terms of dyons coordinates as a determinant of certain matrix $G$, to be referred to as Diakonov determinant. In a dilute limit it leads to Coulomb interactions between the dyons, but in the dense region it becomes strongly repulsive, till at certain density the moduli volume vanishes.

\footnotetext{
${ }^{2}$ They are called "instanton-monopoles" by Unsal et al, and are similar but not identical to "instanton quarks" discussed by Zhitnitsky et al.
} 
Interesting semi-classical description of the confining regime has been found by Unsal and collaborators [21] in a carefully devised setting of softly broken supersymmetric models. While the setting includes a compactification on a small circle, with weak coupling and an exponentially small density of dyons, the minimum at the confining holonomy value is induced by the repulsive interaction in the dyon-antidyon molecules (called bions by these authors). The crucial role of the supersymmetry is the cancellation of the perturbative Gross-Pisarski-Yaffe-Weiss (GPYW) holonomy potential: as a result, in this setting there is no deconfined phase with trivial holonomy at all, unless supersymmetry is softly broken. Sulejmanpasic and myself [18] proposed a simple analytic model for the dyon ensemble with dyon-antidyon "repulsive cores", and have shown how they may naturally induce confinement in dense enough dyonic ensemble.

Traditional explanation of the confinement is often given in terms of particle-monopoles and dual superconductor model of 't Hooft and Mandelstamm: so one may ask about any relation between particle-monopoles and instanton-dyons. In $\mathscr{N}=2$ SYM (Seiberg-Witten theory) both are under theoretical control, so one can calculate how each of them contribute to partition function. Remarcably [22] one finds that the results are equal! One can see that particle-monopoles provide better behaved sum at low- $T$, while the instanton-dyons better converge at the high- $T$ end, but both describe the same physics. Unfortunately, in non-supersymmetric theories, without such explicit expressions available, the relation between the two remains unclear.

\section{Classical dyon-antidyon interaction and the streamlines}

As it is well known, the selfduality of instantons or their constituents, combined with the famous Bogomolny inequality, relates the classical action to the topological charge and thus protect them from classical interactions. Any number of $M, L$ dyons, or $\bar{M}, \bar{L}$ have the same total action, independent on their positions: as a result one has exact moduli spaces. (The interaction only appears at the one-loop order, as we already mentioned above.)

This however is no longer true when one mixes together selfdual and anti-selfdual objects ${ }^{3}$. Instanton-antiinstanton configurations can be mapped via the so called streamline, a one-parameter set of solutions defined by a condition that the driving force (current), while nonzero, is tangent to the set. A practical way to generate them is to follow the gradient flow, starting from some initial ansatz, as was done numerically for the instanton-antiinstanton in the double-well potential in [24].

Let me add a new comment on quantum-mechanical streamline, related with recent renewed interest in "resurgent series". Not going into formulae here, let me just remind that it is based on "three whales", and the coefficients have three indices, corresponding to three basic elements: (i) $g$, the coupling constant, (ii) tunneling series over powers of $\exp (-$ const $/ g)$ where the constant is defined by instantons, and (iii) the powers of $\log (1 / g)$. The first are well known perturbative series, the second is the instanton series: but what is the "third whale"? These terms originate come from the "lower part" of the instanton-antiinstanton streamlines, or from the "III molecules". This streamline goes all the way to trivial configuration, with zero field and action, $S(\Delta t \rightarrow 0)=0$. Yet the semiclassical theory is only valid when the action remains large $S(\Delta t) \sim \exp (-t) / g \gg 1$,

\footnotetext{
${ }^{3} \mathrm{~A}$ famous way to avoid it is to use supersymmetry-based holomorphy arguments, which forbid interaction between the sectors. This nice escape root has been taken by many, but for me dealing with only non-existing toy theories seemed to be too heavy price to pay for mathematical convenience.
} 
which creates nontrivial lower integration limit on $\Delta t>\log (1 / g)$, and thus contribution to the total energy. In gauge theories such terms, coming from the repulsive core in the instanton-antiinstanton channels, are also known, and in fact were the important element of the Interacting Instanton Liquid Model.

For gauge field instantons the first study of the instanton-antiinstanton streamline was done by Balitsky and Yung [25], in the large-distance approximation. Since classical instanton-antiinstanton problem is intrinsically conformal, one can perform a conformal transformation into a co-central configuration, relating the gauge theory and the double-well instantons. Using this method Verbaarschot [26] found a quite accurate analytic approximation to the instanton-antiinstanton streamline.

Larsen and myself have recently calculated the dyon-antidyon $M \bar{M}, L \bar{L}$ streamline [23], by performing gradient flow calculations on a $3 \mathrm{~d}$ lattice. We start with two separated and "well combed" dyons, with $A_{4}$ going into the same direction at all infinities, and then start to minimize the action along the gradient flow direction. Many details can be found in the original paper, the key Fig. 1(a) shows how the action depends on the computer time. The gradient flow process was found to proceed via the following stages:

(i) near initiation: starting from relatively arbitrary ansatz one finds rapid disappearance of artifacts and convergence toward the streamline set

(ii) following the streamline itself. The action decrease at this stage is small and steady. The dyons basically approach each other, with relatively small deformations: thus the concept of an interaction potential between them makes sense at this stage

(iii) a metastable state at the streamline's end: the action remains constant, evolution is very slow and consists of internal deformation of the dyons rather than further approach

(iv) rapid collapse into the perturbative fields plus some (pure gauge) remnants

The existence of the stage (iii) has not been anticipated. All configurations corresponding to it have the same action, and - within our accuracy the same dyon-antidyon distance. One can perhaps lump all of them into a new metastable configuration, perhaps identifiable on the lattice.

Our other comment is that even at the end of the streamline the action value is not that far from the sum of the two dyon masses. In other words, the classical interaction potential appears to be in a sense numerically small, although parametrically dominant: a welcoming feature for statistical mechanics simulations.

Last but not least, we do observe the universality of the streamline. It means that, independently on the initial dyon separation and the details of the initial states, the gradient flow path proceeds through essentially the same set of configurations, at stages (ii-iv). Thus one-parameter characterization of those is possible. A parameter we found most practical in this work is simply its lifetime - duration in our computer time $\tau$, needed for a particular configuration to reach a final collapse. , following through the so called "streamline" set of configurations, till their collapse to perturbative fields.

Finally, let me add that the last $M \bar{L}, L \bar{M}$ channels were also investigated recently. (It was not done in the original paper because in this case the configurations are time-dependent and thus $3 \mathrm{~d}$ lattice was insufficient.) The interaction is repulsive, approximately sign flipped version of that discussed above. 


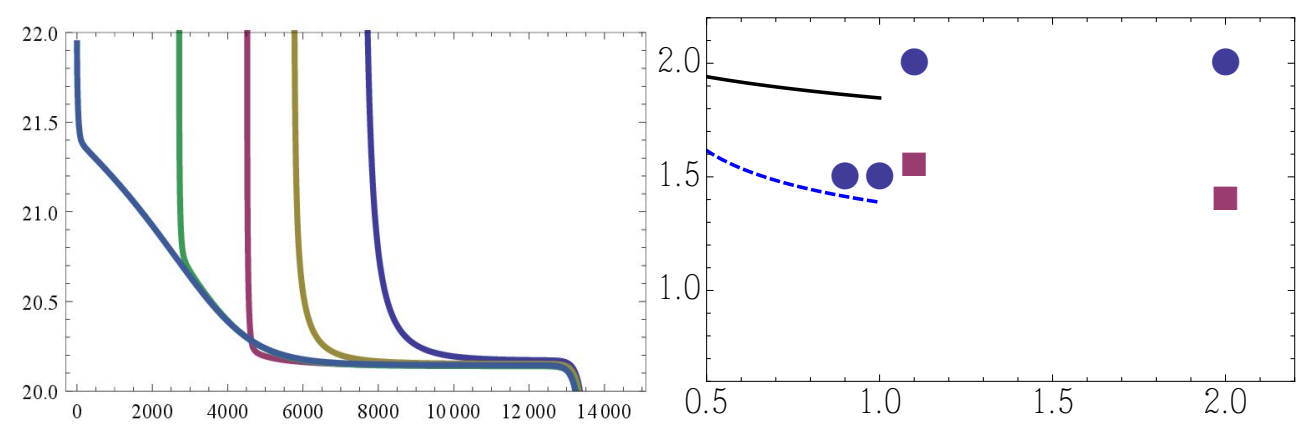

Figure 1: (a) 3 d-action $S_{3}$ for the $M \bar{M}$ dyon pair, as a function of computer time of the gradient flow. Subsequent lines are for separations $\left|r_{M}-r_{\bar{M}}\right| v=0,2.5,5,7.5,10$, from right to left in the graph. The action of two well separated dyons is 23.88. (b) The electric $M_{E} / T$ (dashed line) and magnetic $M_{M} / T$ (solid line) screening masses in versus $T / T_{c}$. The points are SU(2) lattice data from shown for comparison, (blue) circles are $M_{E} / T$, (red) squares are $M_{M} / T$

\section{Dense dyonic plasma}

In this short summary there is no place to discuss numerical parameterizations and other details: so we only explain the key issues of the problem. The classical dyon-antidyon interaction is about an order of magnitude stronger than the one-loop effects studied before. The potential (normalized so that partition function includes $\exp (-V)$ ) is $V \sim O(1)$ and therefore in general it is strong enough to make the dyon plasma to be strongly coupled. Such potential in general generates strong correlations, requiring brute force methods to study them. Those will be discussed at the end of this talk.

However, if the dyon plasma is sufficiently dense, it also generates strong screening effects. Those effectively weaken the interaction, till above certain density it become manageable via the usual Debye-Huckel approximation. For brevity, we call this regime "dense dyon plasma", DDP for short. Its theory has been developed in the paper by Y. Liu, I. Zahed, and myself [27] . The dyonic plasma is dense enough at low temperature. Furthermore, as we found that the holonomy needs to be at the confining value $v=\pi T$, the applicability of this work is restricted to only the confining phase $T<T_{c}$.

While the one-loop effects are subleading in a pairwise interactions at large distances, it turns out that manybody effects of the moduli volume gets strongly repulsive at higher density. Inclusion of the one-loop effect via Diakonov determinant is also included: after some changes of variables we introduce new bosonic fields for both classical and one-loop Coulomb-like forces. The formulae are to be followed in the original paper, as they are quite involved. We found that running coupling induces subtle changes in the interaction: the effective coupling (given by the interaction formfactor at most important momentum) changes sign, which manifests itself directly in crossing of the electric and magnetic screening masses. The results are shown in Fig. 1(b) we display the results for $M_{E, M} / T$ in the range $(0.5-1) T_{c}$ versus $T / T_{c}$.

The points at $T>T_{C}$ are lattice data shown for comparison. Note that the electric mass drops down sharply at $T_{c}$, and, as a result, in the region we study magnetic screening is stronger $M_{M}>M_{E}$, while above $T_{c}$, in a more familiar QGP region, the opposite relation is true, $M_{M}<M_{E}$. This switching of the magnitude of the two screening masses is dynamically explained in the paper. 
Many more results on the DDP properties has been calculated in this approach: the free energy, densities of the dyons, string tension, correlators of various Polyakov and Wilson lines, etc. All of them can and should be compared to lattice studies.

\section{Chiral symmetry breaking and instanton-dyons}

Topological index theorem require one (fundamental) $4 \mathrm{~d}$ fermionic zero mode per unit topological charge. An instanton has $N_{c}$ constituent dyons: and the issue where the fermion mode is located. The question has been answered quickly by van Baal and collaborators: the fermionic zero modes is located primarily at one of the dyons, jumping to another one as a function of holonomy value and/or periodicity phase of the fermions, as certain relation between them is fulfilled. For the simplest color group $S U(2)$ mostly discussed so far, the physical (anti-periodic) quarks have zero mode on $L$ dyons, while the periodic once have zero modes located at $M$ dyons.

Sulejmanpasic and myself [17] have analyzed a number of phenomena induced by the fermionic zero modes of the instanton-dyons such as the formation of clusters (molecules or bions) at high temperature and the spontaneous breaking of chiral symmetry at low temperature. Several puzzles originating from lattice observations have been explained on this way.

For example, quenched simulations of the gauge theory had shown deconfinement at some $T_{c}$. Although no fermions were included in the theory, one can add them as an analysis tool later, and check in particular where chiral symmetry is broken. Quite early it was observed that at $T<T_{c}$ the chiral symmetry is broken, and restored above $T_{c}$, for physical (anti-periodic) fermions. Later it was observed, that using periodic fermions instead one gets a very different quark condensate, which does not disappear at deconfinement and persisted well above $T_{c}$. It was puzzling in the instanton framework, since there is one zero mode per instanton, for both periodic and antiperiodic fermions. It became clear in the dyon setting, which allows different action - and thus density for $M$ and $L$ dyons. Above $T_{c}$ the $M$ dyons are "lighter" than $L$ ones, and more numerous. Periodic fermions "see" the ensemble of $M, \bar{M}$, while anti periodic "see" the ensemble of $L, \bar{L}$ ones: so the difference is natural.

The mean field approach to the problem with fermions is discussed in the second paper of the dense plasma series, of Liu,Zahed and myself [29]. After certain manipulations with fermions, we bosonize the partition function. The scalar mesonic field $\sigma=\bar{q} q$ obtains the corresponding gap equation on its VEV, only slightlyy more complex than in the Nambu-Jona-Lasinio-type models. We explicitly solve it for different number of flavors $N_{f}$ and discuss how that nonzero solution deviates from previous studies with instantons.

\section{Numerical simulations of the dyon ensemble}

The first paper attempting direct Metropolis study of the dyon ensemble was by Faccioli and myself [14]. It has a number of methodical differences from the instanton ensembles: the interactions are long-range and thus more sensitive to boundaries. We decided to put the dyons on a 3-d sphere rather than the tori (used in many other works including lattice gauge theory) with a simplified Coulomb on a sphere. Other methodical details should be found in the paper. 
The one-loop Diakonov determinant generates repulsive interaction at high density: this feature we have studied in detail. However the main thrust of that paper was on fermions and chiral symmetry breaking, as a function of the number of flavors. Generic phenomenon, familiar already from the instanton liquid, is that at high $T$ - low dyon density - one finds clustering of $L \bar{L}$ dyons, while at high density the quasi zero modes get collectivized and the chiral symmetry gets broken. This mechanism is very robust for $N_{f}=1,2,3$ flavors but falters at 4 and perhaps does not work at 5 and more.

As we already mentioned above, the classical dyon-antidyon interactions are strong enough to make the ensemble "strongly coupled", with significant correlations between particles. This interaction has not be included by Faccioli and myself: but its presence makes direct statistical simulations even more necessary.

New series of simulations is now performed by Larsen and myself [28]. Using GPU instead of CPU for evaluation of the determinants (of the Diakonov matrix) had speed up the program quite a bit. The main issue we focus on is the back reaction of the dyons on the holonomy value $v$. We do observe the confinement phase transition, from small $v$ at high $T$ to its confining value below certain $T_{c}$. The part which is more delicate and still needs more work is to self-consist the dyon density and holonomy values. Generalization of this work to theories with fermions is not yet done but is expected to be rather straightforward.

\section{Summary}

After a certain minimum of activity, studies of gauge topology are getting more active again. New round of works on quantum mechanical instantons appeared, focusing on trans-series and relations between the perturbative and instanton series. A nice bridge to supersymmetric models has been developed by Unsal and collaborators.

The semiclassical theory of gauge topology at nonzero temperatures, including nonzero holonomy and thus based on the instanton-dyons, is becoming quantitative. Its applicability region cover approximately the temperature range $0.5<T / T_{c}<2$. At the upper range the ensemble is a dilute gas made of very correlated clusters. At the lower $T$ the system is dense dyonic plasma, DDP, with strong effective screening, allowing to use a Debye-Huckel-like mean field theory.

Unlike the previous topological models, now both nonperturbative phenomena - confinement and chiral symmetry breaking - are explained in the same framework. Confinement is generated by the back reaction of the dyons on the holonomy potential, while the chiral symmetry breaking is induced by collectivization of the fermionic zero modes of some dyon type, similar to that in the instanton liquid models. They both require dense enough ensemble, and at current accuracy we don't separate their effective critical temperatures: perhaps those would be revealed by future more accurate studies. On the lattice, the situation is similar: there are indications to separation of deconfinement and chiral phase transitions at higher $N_{f}$, but the phenomenon is not yet well quantified.

Acknowledgements A lot of credit in this subfield go to our friends who are no longer with us, Pierre van Baal and Dmitri Diakonov. My progress in understanding those issues would not be possible without my collaborators on the projects discussed above, P.Faccioli, I.Zahed, 
T.Sulejmanpasic and R.Larsen. My work was supported by the U.S. Department of Energy under Contracts No. DE-FG-88ER40388.

\section{References}

[1] A. A. Belavin, A. M. Polyakov, A. S. Schwartz and Yu. S. Tyupkin, Phys. Lett. B 59, 85 (1975).

[2] E. V. Shuryak, Nucl. Phys. B 203, 93 (1982).

[3] T. Schafer and E. V. Shuryak, Rev. Mod. Phys. 70, 323 (1998) [arXiv:hep-ph/9610451].

[4] R. Rapp, T. Schafer, E. V. Shuryak and M. Velkovsky, Phys. Rev. Lett. 81, 53 (1998) [hep-ph/9711396].

[5] N. I. Kochelev, Phys. Lett. B 426, 149 (1998) [hep-ph/9610551]; D. Ostrovsky and E. Shuryak, Phys. Rev. D 71, 014037 (2005) [hep-ph/0409253]; Y. Qian and I. Zahed, Phys. Rev. D 86, 014033 (2012) [Erratum-ibid. D 86, 059902 (2012)] [arXiv:1112.4552 [hep-ph]]; Y. Qian and I. Zahed, Phys. Rev. D 90, no. 11, 114012 (2014) [arXiv:1404.6270 [hep-ph]].

[6] N. A. Nekrasov, Adv. Theor. Math. Phys. 7, 831 (2004) [hep-th/0206161].

[7] G. Basar and G. V. Dunne, arXiv:1501.05671 [hep-th].

[8] M. A. Escobar-Ruiz, E. Shuryak and A. V. Turbiner, arXiv:1501.03993 [hep-th].

[9] G. V. Dunne and M. Unsal, Phys. Rev. D 89, no. 10, 105009 (2014) [arXiv:1401.5202 [hep-th]].

[10] L. Y. Glozman, C. B. Lang and M. Schrock, Phys. Rev. D 86, 014507 (2012) [arXiv:1205.4887 [hep-lat]].

[11] T. C. Kraan and P. van Baal, Phys. Lett. B 435, 389 (1998) [arXiv:hep-th/9806034].

[12] K. -M. Lee and C. -h. Lu, Phys. Rev. D 58, 025011 (1998) [hep-th/9802108].

[13] E. Poppitz, T. Schafer and M. Unsal, JHEP 1303, 087 (2013) [arXiv:1212.1238].

[14] P. Faccioli and E. Shuryak, Phys. Rev. D 87, no. 7, 074009 (2013) [arXiv:1301.2523 [hep-ph]].

[15] E. M. Ilgenfritz and E. V. Shuryak, Nucl. Phys. B 319, 511 (1989).

[16] S. Sharma, V. Dick, F. Karsch, E. Laermann and S. Mukherjee, arXiv:1311.3943 [hep-lat].

[17] E. Shuryak and T. Sulejmanpasic, Phys. Rev. D 86, 036001 (2012) [arXiv:1201.5624 [hep-ph]].

[18] E. Shuryak and T. Sulejmanpasic, Phys. Lett. B 726, 257 (2013) [arXiv:1305.0796 [hep-ph]].

[19] D. Diakonov, N. Gromov, V. Petrov and S. Slizovskiy, Phys. Rev. D 70, 036003 (2004) [arXiv:hep-th/0404042].

[20] D. Diakonov, Topology and Confinement, arxiv:0906.2456v1 (2009)

[21] E. Poppitz, T. Schaefer and M. Unsal, JHEP 1210, 115 (2012) [arXiv:1205.0290 [hep-th]].

[22] E. Poppitz and M. Unsal, JHEP 1107, 082 (2011) [arXiv:1105.3969 [hep-th]].

[23] R. Larsen and E. Shuryak, arXiv:1408.6563 [hep-ph].

[24] E. V. Shuryak, Nucl. Phys. B 302, 621 (1988).

[25] I. I. Balitsky and A. V. Yung, Phys. Lett. B 168, 113 (1986).

[26] J. J. M. Verbaarschot, Nucl. Phys. B 362, 33 (1991) [Erratum-ibid. B 386, 236 (1992)]. 
[27] Y. Liu, E. Shuryak and I. Zahed, “Confining Dyon-Anti-Dyon Coulomb Liquid Model I," arXiv:1503.03058 [hep-ph].

[28] R. Larsen and E. Shuryak, "Interacting Ensemble of the Instanton-dyons and Deconfinement Phase Transition in the SU(2) Gauge Theory," arXiv:1504.03341 [hep-ph].

[29] Y. Liu, E. Shuryak and I. Zahed, "Light Quarks in the Screened Dyon-Anti-Dyon Coulomb Liquid Model II," arXiv:1503.09148 [hep-ph]. 